\title{
Inverse Independence Number of a Graph
}

\author{
P.G.Bhat \\ Department of Mathematics \\ Manipal Institute of Technology \\ Manipal, India, 576104
}

\author{
Surekha R Bhat \\ Department of Mathematics \\ Milagres College \\ Kallianpur, Uudpi \\ INDIA , 576107
}

\begin{abstract}
The concept of inverse domination was introduced by Kulli V.R. and Sigarakanti S.C. [9] . Let D be a $\gamma$ - set of G. A dominating set $\mathrm{D}_{1} \subseteq \mathrm{V}$ - D is called an inverse dominating set of $\mathrm{G}$ with respect to $\mathrm{D}$. The inverse domination number $\gamma^{\prime}(\mathrm{G})$ is the order of a smallest inverse dominating set. Motivated by this definition we define another parameter as follows. Let $\mathrm{D}$ be a maximum independent set in G. An independent set $\mathrm{S} \subseteq \mathrm{V}$ - D is called an inverse independent set with respect to D. The inverse independence Number $\beta_{0}{ }^{-1}(G)=\max \{|S|: S$ is an inverse independent set of $\mathrm{G}\}$.We find few bounds on inverse domination number and also initiate the study of the inverse independence number giving few bounds on inverse independence number of a graph.
\end{abstract}

\section{Keywords}

Inverse domination number, Independence number and Inverse Independence number.

\section{INTRODUCTION}

The terminologies and notations used here are as in Harary [5]. By order and size we mean the number of vertices and number of edges respectively in a graph. A set $\mathrm{D} \subseteq \mathrm{V}$ is a dominating set of a graph $\mathrm{G}=(\mathrm{V}, \mathrm{E})$, if every $\mathrm{v} \in \mathrm{V}-\mathrm{D}$ is adjacent to some $\mathrm{u} \in \mathrm{D}$. The domination number $\gamma=\gamma(\mathrm{G})$ is the cardinality of a minimum dominating set of $\mathrm{G}$. This concept is well studied in [6]. A set $\mathrm{D} \subseteq \mathrm{V}$ is said to be independent if no two vertices in $\mathrm{D}$ are adjacent. The independence number $\beta_{0}(G)$ is the maximum cardinality of an independent set of $\mathrm{G}$. We say that an edge $\mathrm{x}$ and a vertex $\mathrm{v}$ cover each other if $\mathrm{x}$ is incident on $\mathrm{v}$. A set $\mathrm{D} \subseteq \mathrm{V}$ is said to be a vertex cover if every edge in $\mathrm{G}$ is covered by some vertex in D. The vertex covering number $\alpha_{0}(G)$ is the cardinality of a minimum vertex cover of G. A minimum vertex cover (maximum independent set) is denoted as $\alpha_{0}-$ set (respectively $\beta_{0}-$ set).

Remark 1.1. A set $S \subseteq V$ is a vertex cover if and only if $\mathrm{V}-\mathrm{S}$ is an independent set.

The independence number and vertex covering number are related by the classical Gallai's Theorem.

Theorem 1.1. [Gallai]. For any graph $\mathrm{G}$,

$$
\alpha_{0}(G)+\beta_{0}(G)=p
$$

\section{BOUNDS ON INVERSE DOMINATION NUMBER}

The concept of inverse domination is introduced by Kulli V.R and Sigarakanti S.C [9]. Let D be a $\gamma$ - set of G. If $\mathrm{D}_{1} \subseteq \mathrm{V}$ - D is a dominating set, then $\mathrm{D}_{1}$ is called the inverse dominating set of $G$ with respect to $D$. The inverse domination number $\gamma^{\prime}(\mathrm{G})$ is the order of a smallest inverse dominating set. If D is a minimal dominating set of an isolate free graph $G$, then $\mathrm{V}-\mathrm{D}$ is also a dominating set of $\mathrm{G}$. Therefore every isolate free graph has an inverse dominating set. Henceforth, by a graph $\mathrm{G}$, we mean an isolate free and simple graph. It is observed that $\gamma(\mathrm{G}) \leq \gamma^{\prime}(\mathrm{G})$ and $\gamma(\mathrm{G})+\gamma^{\prime}(\mathrm{G}) \leq \mathrm{p}$. Domke et.al [3] characterized the graphs for which $\gamma(\mathrm{G})+\gamma^{\prime}(\mathrm{G})=\mathrm{p}$. Tamiz Chelvam and Grace Prema G.S. [10], characterized the graphs for which domination and inverse domination numbers are equal. Ameenal Bibi K. and Selvakumar R. [1] studied the Split and nonsplit inverse domination. They also extended inverse domination to semi-total block graph in [2]. Another parameter called disjoint domination number $\gamma \gamma(\mathrm{G})$ defined by Hedetnimi et.al [7] as $\min \left\{\left|S_{1}\right|+\left|S_{2}\right| ; S_{1}, S_{2}\right.$ are disjoint dominating sets of $\mathrm{G}\}$. They call $\mathrm{G}$ is $\gamma \gamma$-minimum if $\gamma \gamma(\mathrm{G})=$ $2 \gamma(\mathrm{G})$ and $\mathrm{G}$ is $\gamma \gamma$-maximum if $\gamma \gamma(\mathrm{G})=\mathrm{p}$. The following theorem from [6] which gives a lower bound for domination number is used to obtain an upper bound for inverse domination number.

Theorem 2.1[6]. For any (p, q) graph G,

$$
\max \left\{\mathrm{p}-\mathrm{q}, \quad \frac{\mathrm{p}}{1+\Delta}\right\} \leq \gamma
$$

Proposition 2.2. For any $(p, q)$ graph $G$,

$$
\gamma^{\prime} \leq \min \left\{q, \frac{p \Delta}{1+\Delta}\right\}
$$

Further, this bound is sharp.

Proof. Let D be any $\gamma$ - set. Since V- D is an inverse dominating set, we have $\gamma^{\prime} \leq|\mathrm{V}-\mathrm{D}|=\mathrm{p}-\gamma \leq \mathrm{p}-\frac{\mathrm{p}}{1+\Delta}=$ $\frac{\mathrm{p} \Delta}{1+\Delta}$ (using Theorem 2.1). Also $\gamma^{\prime} \leq \mathrm{p}-\gamma \leq \mathrm{p}-(\mathrm{p}-\mathrm{q})=\mathrm{q}$. The bound is sharp for any star graph.

A partition $\mathrm{V}_{1}, \mathrm{~V}_{2}, \ldots \ldots, \mathrm{V}_{\mathrm{d}}$ of the vertex set $\mathrm{V}$ is called a domatic partition of $\mathrm{G}$ if each $\mathrm{V}_{\mathrm{i}}, 1 \leq \mathrm{i} \leq \mathrm{d}$ is a dominating set of $\mathrm{G}$. The domatic number $\mathrm{d}(\mathrm{G})$ is the maximum order of a domatic partition of G. In our next result we obtain a bound for $\gamma^{\prime}$ in terms of domatic number $\mathrm{d}(\mathrm{G})$. 
Proposition 2.3. Let $\mathrm{G}$ be a graph of order $\mathrm{p}$ and domatic number $d(G)$. Let $V_{1}, V_{2}, \ldots . . V_{d}$ be the domatic partition of G such that $\mathrm{V}_{1}$ is a $\gamma$ - set and $\mathrm{V}_{2}$ is a $\gamma^{\prime}$ set of $\mathrm{G}$.

$$
\text { Then } \quad \gamma^{\prime} \leq \frac{\mathrm{p}-\gamma}{\mathrm{d}-1} \text {. }
$$

Further, this bound is sharp.

Proof. Let $V_{1}, V_{2}, \ldots \ldots V_{d}$ be the domatic partition of $G$ satisfying the condition stated in the proposition. Then $\left|\mathrm{V}_{1}\right|+\left|\mathrm{V}_{2}\right|+\ldots \ldots+\quad+\left|\mathrm{v}_{\mathrm{d}}\right|=\mathrm{p}$. Since $\gamma^{\prime} \leq\left|\mathrm{V}_{\mathrm{i}}\right|$, for each $2 \leq \mathrm{i} \leq \mathrm{d}$, we have $(\mathrm{d}-1) \gamma^{\prime} \leq\left|\mathrm{v}_{2}\right|+\ldots \ldots .+\left|\mathrm{v}_{\mathrm{d}}\right|=$ $\mathrm{p}-\left|\mathrm{V}_{1}\right|=\mathrm{p}-\gamma$ and the result follows. The bound is sharp for any star graph $\mathrm{K}_{1, \mathrm{n}}$, complete graph $\mathrm{K}_{\mathrm{p}}$ and cycle $\mathrm{C}_{3 \mathrm{n}}$.

We improve the bound obtained in Proposition 2.3 in the next corollary.

Corollary 2.3.1. Let $\mathrm{G}$ be a graph with $\mathrm{p}$ vertices, and domatic number $\mathrm{d}(\mathrm{G})$. Then

$$
\gamma^{\prime} \leq \frac{\mathrm{p} \Delta}{(1+\Delta)(\mathrm{d}-1)}
$$

Further, this bound is sharp.

Proof. From Proposition 2.2, $\mathrm{p}-\gamma \leq \mathrm{p}-\frac{\mathrm{p}}{1+\Delta}=\frac{\mathrm{p} \Delta}{1+\Delta}$. Using this in Proposition 2.3, we get the desired result. The bound is

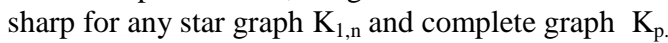

For any cycle $\mathrm{C}_{3 \mathrm{n}}, \gamma^{\prime}\left(\mathrm{C}_{3 \mathrm{n}}\right)=\mathrm{n}$ and $\mathrm{d}\left(\mathrm{C}_{3 \mathrm{n}}\right)=3$. Therefore $\gamma^{\prime}\left(\mathrm{C}_{3 \mathrm{n}}\right)=\mathrm{n}=\frac{3 \mathrm{n} \times 2}{3 \times 2}=\frac{\mathrm{p} \Delta}{(1+\Delta)(\mathrm{d}-1)}$. Thus $\mathrm{C}_{3 \mathrm{n}}$ attains the bound in the Corollary. For the graphs with $\mathrm{d}(\mathrm{G})>2$, the bound given in Corollary 2.3.1 is an improved bound than that given in Proposition 2.2.

Domke et.al [3] proved that for any Tree $\mathrm{T}$ of order $\mathrm{p}$, $\frac{\mathrm{p}+1}{3} \leq \gamma^{\prime}(\mathrm{T})$. Using the same proof technique we now obtain a lower bound for inverse domination number in terms of the size of the graph.

\section{Proposition 2.4.}

For any graph $\mathrm{G}$ of order $\mathrm{p}$, and size $\mathrm{q}$,

$$
\frac{2 \mathrm{p}-\mathrm{q}}{3} \leq \gamma^{\prime}
$$

Proof. Let $D_{1}$ be the $\gamma$ - set and $D_{2}$ be the $\gamma^{\prime}$-set of $G$. Then there are at least $\gamma$ edges from $D_{1}$ to $D_{2}$ and $V-\left(D_{1} \cup D_{2}\right)$ edges from $\mathrm{D}_{1}$ to $\mathrm{V}-\mathrm{D}_{1}$ and $\mathrm{V}-\left(\mathrm{D}_{1} \cup \mathrm{D}_{2}\right)$ edges from $\mathrm{D}_{2}$ to $\mathrm{V}-\mathrm{D}_{2}$. Therefore $\mathrm{q} \geq 2\left(\mathrm{~V}-\left(\mathrm{D}_{1} \cup \mathrm{D}_{2}\right)\right)+\gamma$

$$
\begin{aligned}
& =2\left(\mathrm{p}-\left(\gamma+\gamma^{\prime}\right)\right)+\gamma \\
& =2 \mathrm{p}-\gamma-2 \gamma^{\prime} \geq 2 \mathrm{p}-3 \gamma^{\prime} \quad \text { which yields the }
\end{aligned}
$$

desired bound.

If $G$ is a cycle with $p$ vertices, then $\frac{(2 p-q)}{3}=\frac{(2 p-q)}{3}=\frac{p}{3}=\gamma^{\prime}$. Hence the bound is sharp for any cycle $\mathrm{Cp}$. One can easily observe that bound is also attained for the graph $\mathrm{K}_{4}-\mathrm{x}$ where $\mathrm{x}$ is any edge of the complete graph $\mathrm{K}_{4}$.

Since for any tree $\mathrm{T}, \mathrm{q}=\mathrm{p}-1$, the next result, originally given by Domke et.al [3] follows as a Corollary to the above Proposition.

Corollary 2.4.1. For any Tree $\mathrm{T}$ of order $\mathrm{p}$,

$$
\frac{\mathrm{p}+1}{3} \leq \gamma^{\prime}(\mathrm{T})
$$

Remark 2.1. If $\mathrm{H}$ is any spanning subgraph of $\mathrm{G}$, then $\gamma^{\prime}(G) \leq \gamma^{\prime}(H)$. Based on this observation we have the next result.

Proposition 2.5. If $G$ is Hamiltonian, then $\gamma^{\prime}(G) \leq\left\lceil\frac{p}{3}\right\rceil$

Proof. Let $\mathrm{G}$ be a Hamiltonian graph. Then there exists a Hamiltonian cycle $C_{p}$ which is a spanning subgraph of $G$. Then from the above remark we have $\gamma^{\prime}(G) \leq \gamma^{\prime}\left(C_{p}\right) \leq\left\lceil\frac{p}{3}\right\rceil$.

\section{INVERSE INDEPENDENCE NUMBER}

The concept of inverse dominating sets motivated us to define another parameter as follows. Let $\mathrm{D}$ be a maximum independent set in G. An independent set $\mathrm{S}$ in $\mathrm{V}-\mathrm{D}$ is called an inverse independent set with respect to $D$. The inverse independence Number $\boldsymbol{\beta}_{\mathbf{0}}^{\mathbf{- 1}}(\mathbf{G})$ is the order of the largest inverse independent set of $\mathrm{G}$. We initiate the study of the new parameter and obtain few bounds on inverse independence number of a graph.

Remark 2.1. If $\mathrm{S} \subseteq \mathrm{V}$, then the subgraph induced by the set $\mathrm{S}$ is denoted as $\langle\mathrm{S}\rangle$. Infact, any inverse independent set of $\mathrm{G}$ is a maximal independent set of $\langle V-D\rangle$, the subgraph induced by the set V-D where $\mathrm{D}$ is a $\beta_{0}$-set of $\mathrm{G}$. This fact confirms the existence of an inverse independent set for any graph $\mathrm{G}$. Therefore $\beta_{0}^{-1}(G)=\beta_{0}\langle\mathrm{~V}-\mathrm{D}\rangle$.

Another variety of independence number called strong independence number introduced by S.S.Kamth and R.S.Bhat [8]. A vertex $v$ is said to be strong if $d(v) \geq d(u)$ for every $u$ adjacent to v. An independent set in which every vertex is strong is called a strong independent set. Several properties of strong independent sets are studied in [8]

\subsection{Inverse Covering Number}

Let $\mathrm{D}$ be a minimum vertex covering of $\mathrm{G}$. A set $\mathrm{S} \subseteq \mathrm{V}$ - D which is a covering of $G$ is called an inverse covering of $G$ with respect to the covering $D$. Then the Inverse Covering number $\alpha_{0}^{-1}(G)$ is the order of smallest inverse covering of G. Note that $\alpha_{0}^{-1}(G)$ need not exist for every graph, for if $D$ is a covering of $G$ then $V-D$ need not be a covering of $G$. For example the complete graph $K_{p}$ does not have any inverse covering. A graph $\mathrm{G}$ is said to be Invertible if $\mathrm{G}$ admits an inverse vertex covering.

\section{Example 3.1.}

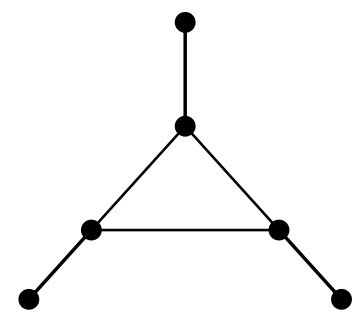

Fig.1.The Corona $\mathrm{K}_{3} \cdot \mathrm{K}_{1}$

The corona of two graphs $G_{1}$ and $G_{2}$ (as defined by Harary [4]) is the graph $G=G_{1} \circ G_{2}$ formed from one copy of $G_{1}$ and $\left|V\left(G_{1}\right)\right|$ copies of $G_{2}$, where the $i^{\text {th }}$ vertex of $G_{1}$ is adjacent to every vertex in the $i^{\text {th }}$ copy of $G_{2}$. For example, the Corona $\mathrm{K}_{3} \cdot \mathrm{K}_{1}$ is shown in the Fig. 1 .

It is well known that every maximal independent set is a minimal dominating set in any graph. But $\beta_{0}^{-1}$ - set need not be a dominating set. For example for the Corona, $\beta_{0}\left(K_{3} \cdot K_{1}\right)=3$ and $\beta_{0}^{-1}\left(K_{3} \cdot K_{1}\right)=2$ and any one pendent vertex together with a nonadjacent nonpendent vertex 
forms a $\beta_{0}^{-1}-$ set which is not a dominating set. Further $\alpha_{0}^{-1}$ does not exists for this graph. Therefore $K_{3} \cdot K_{1}$ is not an invertible graph, whereas every complete bipartite graph $\mathrm{K}_{\mathrm{m}, \mathrm{n}}$ is invertible.

At the outset we give inverse independence number and inverse covering numbers of some standard graphs without proof.

\section{Proposition 3.1.}

(i) For any Path $P_{n}$ and Cycle $C_{n}$ with $n$ vertices

$$
\begin{array}{ll} 
& \beta_{0}^{-1}\left(P_{n}\right)=\left\lfloor\frac{n}{2}\right\rfloor=\beta_{0}^{-1}\left(C_{n}\right) \\
\text { and } & \alpha_{0}^{-1}\left(P_{n}\right)=\left\lceil\frac{n}{2}\right\rceil \\
& \alpha_{0}^{-1}\left(C_{n}\right)=\frac{n}{2} \text { if } n \text { is even } \\
\text { and } \quad C_{n} \text { is not invertible if } n \text { is odd. }
\end{array}
$$

(ii) For any complete graph $\mathrm{K}_{\mathrm{n}}$,

$$
\beta_{0}^{-1}\left(\mathrm{~K}_{\mathrm{n}}\right)=1
$$

and $\quad K_{n}$ is not invertible for any $n$.

(iii) For any complete bipartite graph $\mathrm{K}_{\mathrm{m}, \mathrm{n}}$

$$
\begin{gathered}
\beta_{0}^{-1}\left(K_{m, n}\right)=\min (m, n) \\
\alpha_{0}^{-1}\left(K_{m, n}\right)=\max (m, n)
\end{gathered}
$$

(iv) For any wheel graph $\mathrm{W}_{\mathrm{n}}$

$$
\beta_{0}^{-1}\left(W_{n}\right)=\beta_{0}^{-1}\left(C_{n-1}\right)=\left\lfloor\frac{n-1}{2}\right\rfloor
$$

and $\mathrm{W}_{\mathrm{n}}$ is not invertible for any $\mathrm{n}$.

We now give a condition for $\mathrm{G}$ to be invertible.

Proposition 3.2. Any graph $G$ is invertible if and only if there exists an $\alpha_{0}$-set of $G$ which is independent.

Proof. Suppose there exists an $\alpha_{0}$-set D of $G$ which is independent. Then from Remark 1.1, V-D is an inverse vertex cover. Hence $\alpha_{0}^{-1}(G)$ exists which implies $G$ is invertible. Conversely, suppose $\mathrm{G}$ is invertible. Then $\alpha_{0}^{-1}(\mathrm{G})$ exists and $\mathrm{S}$ be the $\alpha_{0}^{-1}$-set. Let $S_{1}$ be the corresponding $\alpha_{0}$-set of $\mathrm{G}$. We claim that $S_{1}$ is independent. Suppose $S_{1}$ is not independent. Then there exists atleast one edge $\mathrm{x}$ in $\left\langle\mathrm{S}_{1}\right\rangle$. But then the edge $x$ is not covered by any vertex in $S$ and hence $S$ is not a vertex cover of $\mathrm{G}$ - a contradiction. Hence our claim. Thus $S_{1}$ is an $\alpha_{0}$-set which is independent.

In the next theorem we characterize the invertible graphs.

Theorem 3.3. A graph $G$ is invertible if and only if $\alpha_{0}^{-1}(G)+\beta_{0}^{-1}(G)=p$

Proof. Suppose G is invertible. Then by Proposition 3.2, there exists an $\alpha_{0}$-set $\mathrm{D}$ which is independent. Then by Remark 1.1, $\mathrm{V}$ - D is an inverse vertex cover of G. Since no proper subset of $\mathrm{V}-\mathrm{D}$ is a vertex cover, we have $\alpha_{0}^{-1}=|\mathrm{V}-\mathrm{D}|=\mathrm{p}-\alpha_{0}$. Again, since $D$ is independent $\alpha_{0}$-set we have V-D is a $\beta_{0}$-set of $\mathrm{G}$. Hence $\mathrm{D}$ is an inverse independent set. Therefore $|D|=\alpha_{0}=\beta_{0}^{-1}$. Thus $\alpha_{0}^{-1}=\mathrm{p}-\alpha_{0}=\mathrm{p}-\beta_{0}^{-1}$ which yields $\alpha_{0}^{-1}(G)+\beta_{0}^{-1}(G)=p$.

Corollary 3.3.1. If $G$ is invertible, then

$$
\alpha_{0}(G)=\beta_{0}^{-1}(G) \text { and } \beta_{0}(G)=\alpha_{0}^{-1}(G) \text {. }
$$

\section{BOUNDS ON INVERSE INDEPENDENCE NUMBER}

Proposition 4.1. For any graph $\mathrm{G}$ with $\mathrm{p}$ vertices

$$
\begin{array}{ll}
\text { (i) } & \beta_{0}^{-1} \leq \alpha_{0} . \\
\text { (ii) } & \beta_{0}^{-1} \leq \beta_{0} \\
\text { (iii) } & \beta_{0}^{-1} \leq \frac{\mathrm{p}}{2}
\end{array}
$$

Proof. Let $D$ be any $\beta_{0}^{-1}$ set of $G$. If $S$ is any $\beta_{0}$ set of $G$ then $\mathrm{S}$ is a maximum independent set of $\mathrm{G}$. Since $\mathrm{D}$ is independent, we have $\beta_{0}^{-1}=|D| \leq|S|=\beta_{0}$. By definition of inverse independence number $S \cap D=\emptyset$. Also V-S is $\alpha_{0}$-set and $\mathrm{S} \cap \mathrm{D}=\varnothing$ together imply that $\mathrm{D} \subseteq \mathrm{V}$-S. Thus we have $\beta_{0}^{-1}=|\mathrm{D}| \leq|\mathrm{V}-\mathrm{S}|=\alpha_{0}$. Now adding (i) and (ii) and using Theorem 1.1 we get (iii).

Proposition 4.2. Let $\mathrm{G}$ be a $(\mathrm{p}, \mathrm{q})$ graph with minimum degree $\delta$ then $\beta_{0}^{-1} \leq \mathrm{p}-\delta$.

Proof. It is well known that $\delta \leq \alpha_{0}$. Then $\beta_{0}^{-1} \leq \beta_{0}=\mathrm{p}-$ $\alpha_{0} \leq \mathrm{p}-\delta$ and the result follows. The bound is sharp for $\mathrm{K}_{\mathrm{n}}$, complete graph with $n$ vertices and $K_{n, n}$, n-regular complete bipartite graphs with $2 \mathrm{n}$ vertices.

We improve the above bound under certain conditions.

Proposition 4.3. Let $\mathrm{G}$ be a $(\mathrm{p}, \mathrm{q})$ graph with maximum degree $\Delta$ and $G$ has a $\beta_{0}^{-1}$ set containing atleast one maximum degree vertex. Then $\beta_{0}^{-1} \leq \mathrm{p}-\Delta$.

Proof. Let $\mathrm{D}$ be a $\beta_{0}^{-1}$ set containing atleast one maximum degree vertex and $S$ be the set of all vertices with maximum degree. Then $S \cap D \neq \varnothing$. Let $v \in S \cap D$. Then $v$ is a maximum degree vertex in $\mathrm{D}$ and $\mathrm{D}$ is independent together imply that $\mathrm{D} \subseteq \mathrm{V}-\mathrm{N}(\mathrm{v})$. Therefore $\beta_{0}^{-1}=|\mathrm{D}| \leq|\mathrm{V}-\mathrm{N}(\mathrm{v})|=\mathrm{p}-\Delta$

\section{Example 4.1}

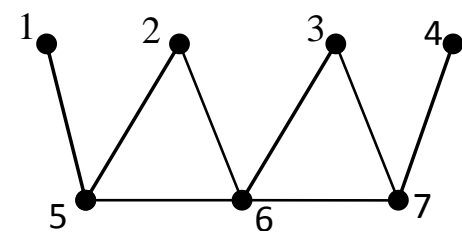

Fig.2

For example for

graph, $\mathrm{K}_{\mathrm{m}, \mathrm{n}}$, $\beta_{0}^{-1}\left(\mathrm{~K}_{\mathrm{m}, \mathrm{n}}\right)=\mathrm{n}=\mathrm{p}-\mathrm{m}=\mathrm{p}-\Delta, \quad$ if $\mathrm{n} \leq \mathrm{m}$. Note that in this case $\beta_{0}^{-1}$-set contains all the maximum degree vertices of $\mathrm{K}_{\mathrm{m}, \mathrm{n} . \mathrm{.}}$ For the graph shown in the Fig $2, \mathrm{~S}=\{1,2,3,4\}$ is a $\beta_{0}$ set. $\mathrm{D}=\{5,7\}$ is a $\beta_{0}^{-1}$-set. But $\mathrm{D}$ does not contain any vertex of maximum degree vertex. $\beta_{0}^{-1}=2<3=\mathrm{p}-\Delta$. Therefore it seems that $\beta_{0}^{-1} \leq \mathrm{p}-\Delta$ for any graph but we could not either prove the result or give a counter example. Hence we put it as an open problem.

Conjecture 4.4. Let $\mathrm{G}$ be a $(p, q)$ graph with maximum degree $\Delta$, then $\beta_{0}^{-1} \leq \mathrm{p}-\Delta$.

Proposition 4.5. Let $\mathrm{G}$ be a $(p, q)$ graph with maximum degree $\Delta$, then $\beta_{0}^{-1} \leq \frac{\mathrm{p} \Delta-\mathrm{q}}{\Delta}$. 
Proof. Since a vertex can cover at most $\Delta$ edges and we need at least $\frac{q}{\Delta}$ edges to cover all the edges of G. Therefore $\frac{q}{\Delta} \leq \alpha_{0}$. Then $\beta_{0}^{-1} \leq \beta_{0}=p-\alpha_{0} \leq p-\frac{q}{\Delta}$ and the result follows. The bound is sharp for any regular complete bipartite graph.

The chromatic number $\chi(G)$ is the minimum number of colours required to colour the vertices of a graph $\mathrm{G}$ such that no two adjacent vertices have same colour. If $\chi(G)=\mathrm{k}$, then the vertex set of $G$ is partitioned in to $k$ independent sets. Next proposition gives a bound for inverse independence number in terms of vertex covering and chromatic number.

Proposition 4.6. Let $\mathrm{G}$ be a $(p, q)$ graph with chromatic number $\chi(G)$ and vertex covering number $\alpha_{0}(G)$. Let $V_{1}, V_{2}, V_{3}, \ldots, V_{k}$ be the partition of the vertex set $V$ in to $k$ independent sets such that $V_{1}$ is a $\beta_{0}$ set and $V_{2}$ is a $\beta_{0}^{-1}$ set of G. Then $\frac{\alpha_{0}}{\chi-1} \leq \beta_{0}^{-1}$. Further this bound is sharp.

Proof. Let $\chi(G)=k$. Suppose that $V_{1}, V_{2}, V_{3}, \ldots, V_{k}$ be the partition of the vertex set $\mathrm{V}$ in to $\mathrm{k}$ independent sets satisfying the condition of the stated in the proposition. Then

$\left|V_{2}\right|+\left|V_{3}\right|+\cdots+\left|V_{k}\right|=p-\left|V_{1}\right|$. Therefore

$\mathrm{p}-\beta_{0}=\alpha_{0}=\left|\mathrm{V}_{2}\right|+\left|\mathrm{V}_{3}\right|+\cdots+\left|\mathrm{V}_{\mathrm{k}}\right| \leq(\mathrm{k}-1) \beta_{0}^{-1}$ and hence the result follows. The bound is sharp for complete graphs and complete bipartite graphs.

The next proposition gives an upper bound for the number of edges when independence number and inverse independence numbers are known

Proposition 4.7. Let $\mathrm{G}$ be a $(\mathrm{p}, \mathrm{q})$ graph with $\beta_{0}=\mathrm{k}$ and $\beta_{0}^{-1}=\mathrm{s}$. Then

$$
\mathrm{q} \leq \frac{1}{2}\left(\mathrm{p}^{2}-\mathrm{k}^{2}-\mathrm{s}^{2}-(\mathrm{p}-\mathrm{k}-\mathrm{s})\right) .
$$

Proof. For any $(\mathrm{p}, \mathrm{q})$ graph $\mathrm{G}, \mathrm{q} \leq\left(\begin{array}{l}\mathrm{p} \\ 2\end{array}\right)$. Further, since $\beta_{0}=\mathrm{k}$ and $\beta_{0}^{-1}=\mathrm{s}$ there cannot exist $\left(\begin{array}{l}\mathrm{k} \\ 2\end{array}\right)+\left(\begin{array}{l}\mathrm{s} \\ 2\end{array}\right)$ edges in $\mathrm{G}$. This implies $\mathrm{q} \leq\left(\begin{array}{l}\mathrm{p} \\ 2\end{array}\right)-\left(\begin{array}{l}\mathrm{k} \\ 2\end{array}\right)-\left(\begin{array}{l}\mathrm{s} \\ 2\end{array}\right)$

$$
=\frac{1}{2}\left(\mathrm{p}^{2}-\mathrm{k}^{2}-\mathrm{s}^{2}-(\mathrm{p}-\mathrm{k}-\mathrm{s})\right) \text {. }
$$

Corollary 4.7.1. Let $\mathrm{G}$ be a (p, $\mathrm{q})$ graph with $\beta_{0}=\mathrm{k}$ and and $\beta_{0}^{-1}=\mathrm{s}$. Then

$$
\beta_{0}^{-1} \leq \frac{1}{2}+\sqrt{\frac{1}{4}+[p(p-1)-k(k-1)-2 q]}
$$

Proof. Suppose that $\beta_{0}^{-1}=$ s. Then from Proposition 4.7, we have $\mathrm{q} \leq\left(\begin{array}{l}\mathrm{p} \\ 2\end{array}\right)-\left(\begin{array}{c}\mathrm{k} \\ 2\end{array}\right)-\left(\begin{array}{c}\mathrm{s} \\ 2\end{array}\right)$.

Hence $2 \mathrm{q} \leq \mathrm{p}(\mathrm{p}-1)-\mathrm{k}(\mathrm{k}-1)-\mathrm{s}(\mathrm{s}-1)$. This yields $s^{2}-s-[p(p-1)-k(k-1)-2 q] \leq 0$. Solving this quadratic equation we get

$$
\begin{aligned}
\mathrm{S} & \leq \frac{1+\sqrt{1+4[\mathrm{p}(\mathrm{p}-1)-\mathrm{k}(\mathrm{k}-1)-2 \mathrm{q}]}}{2} \\
& =\frac{1+2 \sqrt{\frac{1}{4}+[\mathrm{p}(\mathrm{p}-1)-\mathrm{k}(\mathrm{k}-1)-2 \mathrm{q}]}}{2} \\
& =\frac{1}{2}+\sqrt{\frac{1}{4}+[\mathrm{p}(\mathrm{p}-1)-\mathrm{k}(\mathrm{k}-1)-2 \mathrm{q}]} .
\end{aligned}
$$

On the similar lines in the next corollary we get a bound for independence number of a graph when the inverse independence number is known.

Corollary 4.7.2. Let $\mathrm{G}$ be a $(\mathrm{p}, \mathrm{q})$ graph with $\beta_{0}^{-1}=\mathrm{s}$. Then

$$
\beta_{0} \leq \frac{1}{2}+\sqrt{\frac{1}{4}+[p(p-1)-s(s-1)-2 q]}
$$

\subsection{Tripartite Split Graph.}

A graph $\mathrm{G}$ is said to be tripartite split graph if the vertex set of $\mathrm{G}$ can be partitioned in to three subsets $\mathrm{V}_{1}, \mathrm{~V}_{2}, \mathrm{~V}_{3}$ such that $\mathrm{V}_{1}$ and $V_{2}$ are independent and $\left\langle V_{3}\right\rangle$ is complete. For example, the tripartite split graph shown in the Fig.3, attains the bound in Proposition 4.7. For this graph, $\beta_{0}=3, \beta_{0}^{-1}=2, p=7$. Therefore $\mathrm{q}=21-3-1=17=\left(\begin{array}{l}7 \\ 2\end{array}\right)-\left(\begin{array}{l}3 \\ 2\end{array}\right)-\left(\begin{array}{l}2 \\ 2\end{array}\right)=\left(\begin{array}{l}\mathrm{p} \\ 2\end{array}\right)-$ $\left(\begin{array}{l}3 \\ 2\end{array}\right)-\left(\begin{array}{l}2 \\ 2\end{array}\right)$.

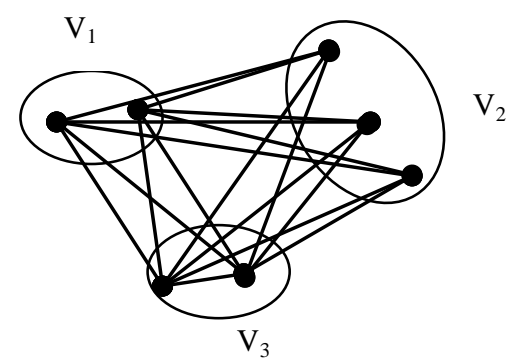

Fig 3. A Tripartite split graph

Similarly, the bound in Corollary 4.7.1 and 4.7.2 are attained for any tripartite split graph.

Again for the graph shown in Fig.3, we have

$$
\begin{aligned}
& \beta_{0}=3= \frac{1}{2}+\sqrt{\frac{1}{4}+[7(7-1)-2(2-1)-2 \times 17]} \\
&=\frac{1}{2}+\sqrt{\frac{1}{4}+[p(p-1)-s(s-1)-2 q]} . \\
& \text { And } \beta_{0}^{-1}=2=\frac{1}{2}+\sqrt{\frac{1}{4}+[7(7-1)-3(3-1)-2 \times 17]} \\
&=\frac{1}{2}+\sqrt{\frac{1}{4}+[p(p-1)-k(k-1)-2 q]}
\end{aligned}
$$

\subsection{Characterization Of Graphs Attaining The Bound In Proposition 4.7.}

One would ask which graphs will attain the bound in Proposition 4.7? This question is answered in our next result. Proposition 4.8. Let $G$ be a connected $(p, q)$ graph with $\beta_{0}=\mathrm{k}$ and $\beta_{0}^{-1}=\mathrm{s}$. Then

$\mathrm{q}=\frac{1}{2}\left(\mathrm{p}^{2}-\mathrm{k}^{2}-\mathrm{s}^{2}-(\mathrm{p}-\mathrm{k}-\mathrm{s})\right)$ if and only if $\mathrm{G}$ is a tripartite split graph.

Proof. If $\mathrm{G}$ is a tripartite split graph with $\beta_{0}=\mathrm{k}$ and $\beta_{0}^{-1}=\mathrm{s}$ it is not hard to verify that $\mathrm{q}=\frac{1}{2}\left(\mathrm{p}^{2}-\mathrm{k}^{2}-\mathrm{s}^{2}-\right.$ $(p-k-s))$. Conversely, suppose $G$ is a graph with $\beta_{0}=k$ 
and $\beta_{0}^{-1}=\mathrm{s}$ and $\mathrm{q}=\frac{1}{2}\left(\mathrm{p}^{2}-\mathrm{k}^{2}-\mathrm{s}^{2}-(\mathrm{p}-\mathrm{k}-\mathrm{s})\right)$. Let $\mathrm{V}_{1}$ be the $\beta_{0}$-set and $V_{2}$ be the $\beta_{0}^{-1}$-set and $V_{3}=V-V_{1}-V_{2}$. Since $\mathrm{V}_{1}$ and $\mathrm{V}_{2}$ are independent and $\mathrm{q}=\frac{1}{2}\left(\mathrm{p}^{2}-\mathrm{k}^{2}-\mathrm{s}^{2}-\right.$ $(\mathrm{p}-\mathrm{k}-\mathrm{s})), \mathrm{G}$ has maximum number of edges. Therefore $\left\langle\mathrm{V}_{3}\right\rangle$ must be complete. Hence $\mathrm{G}$ is a tripartite split graph.

\section{CONCLUSION}

Kulli V.R and Sigarakanthi S.C [9] and Ameenal Bibi K and Salvakumar R [1] quoted some application of inverse domination. Graph theory serves as a model for any binary relation. In domination, both dominating sets and their inverses have important roles to play. Whenever, D is a dominating set, V-D is also a dominating set whenever $\mathrm{G}$ is an isolate free graph. In an information retrieval system, we always have a set of primary nodes to pass on the information. In case, the system fails, we have another set of secondary nodes, to do the job in the complement. When the complement set is connected, then there will be flow of information among the members of the complement. Thus, the dominating sets and the elements in the inverse dominating sets can stand together to facilitate the communication process. Similarly if we want to get an independent set in the compliment set we look for inverse independent set of $\mathrm{G}$. They play very vital role in coding theory, computer science, operations research, switching circuits, electrical networks etc.

\section{REFERENCES}

[1] Ameenal Bibi K., Selvakumar R., The Inverse Split and Non-split Domination in Graphs, IJCA Vol.8- No.7, ( 2010), 21-29.

[2] Ameenal Bibi K, Selvakumar R, The Inverse Domination in Semitotal Block Graphs, IJCA Vol.8- No.8, ( 2010), 4-7.

[3] Domke G.S, Dunbar J.E. and Lisa S Markus, Inverse Dominating sets, Ars Combinatoria, Vol. 72 (2004), 113-118.

[4] Frucht R and Harary F., On Corona of two graphs, Aequationes Math.,4(1970), 322- 324.

[5] Harary F., Graph Theory, Addison Wiley, 1969.

[6] Haynes T.W., Hedetniemi S.T., Slater P.J., Fundamentals of Domination in Graphs, Marcel Dekker, Inc., N.Y., 1998.

[7] Hedetniemi S.M., Hedetniemi S.T, Renu C.Laskar, Slater P.J., Disjoint dominating sets, Proceedings of Int. conf. on Disc. Math. (ICDM 2006) 88- 102.

[8] Kamath S.S. and Bhat R.S., On strong (weak) independence number and vertex coverings of a graph, Discrete Mathematics, 307,(2007) 1136 - 1145.

[9] Kulli V.R. and Sigarakanti S.C., Inverse Domination in Graphs, National Academy science letters, 14 (1991),473-475.

[10] Tamizh Chelvam T and Grace Prema G.S., Equality of Domination and Inverse Domination, ARS Combinatoria, 95(2010), 103-111 